\title{
Quand l'auteur se livre : du vécu à la création littéraire Notes d'une musique ancienne de Salah Benlabed
}

\begin{abstract}
:
This present article is a modest work presentation and synthesis of theoretical concepts related to the issue of identity generic text. The work on which was our Master thesis was full of clues and fictional references that have enabled us to establish a double interpretation, it is therefore in the border between autobiography autofiction. The above article includes all the definitions established by theorists who were interested in this generic. By summoning and highlighting illustrations from our corpus, we were able to identify the characteristics of autobiography and self-fiction, to apply them to our corpus. The latter, analyzed and commented, gave us the image of a very rich and interesting work, given its generic dual membership ; This broadens its outlook reading and highlights its originality.
\end{abstract}

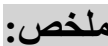

هذه المادة هو عرض العمل المتواضع وتجميع المفاهيم

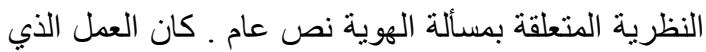

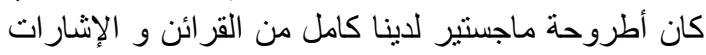

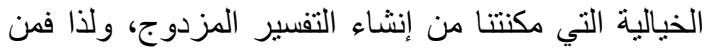

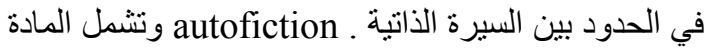
الدذكورة أعلاه كل التعاريف التيى وضعتها التنظية التطرين الذين

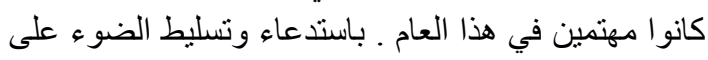

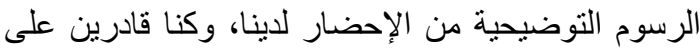

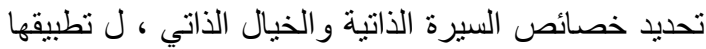

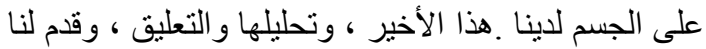

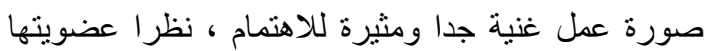

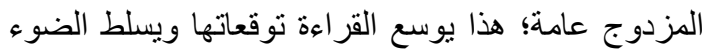

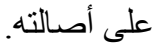

\section{Bendjelloul Lina}

Département de Française

Université des Frères Mentouri

Constantine

\section{Introduction : \\ L’écriture littéraire est une}

véritable expérience, une réelle expédition vers l'imaginaire à travers laquelle un écrivain met en scène une histoire souvent fictive, imaginée qui en plus de sa charge fictionnelle révèle généralement un peu de sa personnalité voire même de son vécu. 
En effet, même si l'on est tenté de croire qu'une telle histoire puisse être le produit de l'inventivité de l'auteur, force est de constater que plus souvent qu'il n'y parait, l'on tombe littéralement sur ce qu'on peut appeler des « confidences » de la part de l'auteur, voire même des confessions, que nous tenterons inconsciemment de mettre en rapport avec certaines références biographiques. À partir du moment où cette comparaison sera formulée et établie, le lecteur fera immersion dans le monde des écritures intimistes.

Les écritures de soi sont, en quelque sorte, des reproductions plus ou moins « respectueuses », selon les fantaisies de l'auteur, de son expérience, de son parcours, plus globalement de sa vie. C'est ce qui s'avère être, ce qu'appellent communément les critiques littéraires, une autobiographie. Pour illustrer ce type d'écriture, nous prendrons comme exemple Notes d'une musique ancienne de l'écrivain Salah Benlabed.Ce récit, qui se voudrait à première vue autobiographique, relate l'histoire d'un reporter qui s'est vu dans l'obligation de s' expatrier, fuyant son pays livré aux intégristes et voulant avant tout sauver sa fille de l'emprise du fanatisme, et qui se retrouve dans un pays dont il ignore tout et auquel il n'arrive pas à s'habituer ; sa terre natale lui manque ainsi que tout ce qui s'y rattache. Sous l'effet de la solitude et de la morosité, les souvenirs d'antan affluent pour redonner goût à un présent lourd de regret et de déconvenue.

\section{Développement}

Pour essayer de comprendre toute l'importance de ce type d'écriture,il serait judicieux d'en donner une définition. Une autobiographie est un récit de vie, ponctué des faits et évènements les plus marquants, aussi heureux ou fâcheux soient-ils, qu'une personne puisse vivre. Aussi loin que l'on puisse remonter, c'est dans la seconde partie du XVIIIe siècle qu'une autobiographie, à proprement parler, a vu le jour. En effet, Les Confessions de Jean-Jacques Rousseau est considéré comme le texte fondateur dans l'écriture autobiographique.

Philippe Lejeune dans son ouvrage Le Pacte autobiographique la définit comme suit: "Récit rétrospectif en prose qu'une personne réelle fait de sa propre existence, lorsqu'elle met l'accent sur sa vie individuelle, en particulier sur l'histoire de sa personnalité. " ${ }^{(1)}$

Cette définition réunit trois critères que doive remplir une autobiographie, à savoir : un récit rétrospectif, en prose, la vie individuelle.

-Récit rétrospectif: l'autobiographie étant un récit de toute une vie, il est quasiment impossible de faire abstraction d'un travail mnémonique, autrement dit, un travail rétrospectif (de retour en arrière) où il sera question de revivre et de convoquer son passé de manière à revoir et ordonner tous les souvenirs qui s'y rattachent. 


\section{Quand l'auteur se livre : du vécu à la création littéraire Notes d'une musique ancienne de Salah Benlabed}

-En prose : la forme d'une autobiographie ne doit pas obéir aux règles et à la rythmique de la versification. Son écriture doit se faire de manière fluide, limpide qui devrait faciliter sa compréhension, une autobiographie en vers n'offre donc pas l'intelligibilité propre à la prose, cette sorte d'irréflexion d'une écriture « désinvolte».

-Vie individuelle: l'autobiographie doit s'attacher à retranscrire, le plus fidèlement possible, la vie d'une personne. Il s'agit de retracer l'évolution de l'individu qu'est l'écrivain. Sa progression de l'être qu'il était (l'enfance, la jeunesse...) vers l'être qu'il est (personne reconnue) est donc capitale. Il sera question de la genèse de sa personnalité et de sa construction.

D'autres spécificités de l'autobiographie ont été relevées à partir des travaux de Philippe Lejeune.

Dans une autobiographie, l'auteur selon Philippe Lejeune doit instaurer, ce qu'il nomme, « un pacte autobiographique ».Ce dernier est, en quelque sorte, un pacte de sincérité dans lequel l'auteur assure au lecteur que ce qu'il avance est pure vérité, qu'il est sincère dans ses propos, c'est un engagement que prend l'auteur de dire sa vie, de la raconter dans un pur esprit de vérité.

L'autobiographie formelle doit réunir dans le pronom personnel «je» trois entités : l'auteur, le narrateur et le personnage. Ce trait caractéristique commun est à l'origine de l'identité onomastique présente dans l'incipit, autrement dit, ce « je » assure plus ou moins la véracité de l'histoire que relate l'auteur et définit par conséquent l'identité générique du texte qui ne peut être qu'une autobiographie. Cette identité onomastique étroitement liée avec l'identité générique du texte peut être révélée au lecteur de deux façons différentes :

-par l'emploi d'un titre sans ambiguités (Les Confessions de Jean-Jacques Rousseau), ou plus simplement par l'emploi du sous-titre « autobiographie ».

-par l'engagement de l'auteur auprès du lecteur en donnant directement son nom au personnage-narrateur (René de François-René de Chateaubriand).De cette façon, l'auteur assure et assume un lien direct avec le personnagenarrateur, et avoue à demi mot la nature autobiographique de son texte.

Néanmoins, et même si l'on est tenté de croire, à travers ces deux démarches l'authenticité du texte, et ainsi de le classer parmi les autobiographies reconnues comme telles, théoriquement parlant, cette conclusion serait hâtive et précipitée. En effet, le titre, le sous-titre ou le fait que le personnagenarrateur porte le même nom que l'auteur ne suffit nullement pour établir l'identité générique du texte, car un récit sous-titré «autobiographie» peut comporter autant d'inventivité et de fiction que dans un texte purement fictionnel.

Dans ce genre de situation, il serait judicieux de repérer et d'analyser ce que Philippe Gasparini nomme «les opérateurs d'identification du héros avec l'auteur » qu'il définit comme suit : "Pourquoi ne pas admettre qu'il existe, outre les nom et prénom, toute une série d'opérateurs d'identification du héros 
avec l'auteur: leur âge, leur milieu socioculturel, leur profession, leurs aspirations, etc. $\|^{(2)}$

Ces indices subtilement insérés dans le texte peuvent être d'éventuelles ressemblances ou points en commun entre le personnage-narrateur (héros) et l'auteur, qu'il faudra toutefois, par la suite, vérifier en établissant une sorte de grille comparative qui engloberait les références biographiques de l'auteur et celles citées dans le texte concernant le personnage ; à partir de là, le lecteur déterminera de façon définitive l'identité générique du récit.

$\mathrm{Si}$, au contraire, cette identification ne se solde pas sur une autobiographie; c'est qu'il s'agit d'une autofiction. Cette dernière résulte de la jonction entre autobiographie et fictionnalité.

Un auteur peut recourir à l'autofiction pour différentes raisons. La première constitue une mesure de protection personnelle; en effet, tous les écrivains ne partagent pas forcément le même besoin ou la même envie de se dévoiler, de se raconter ouvertement. D'aucun préfèrent le faire de manière subtile en fictionnalisant leurs vies, en jouant sur cette oscillation entre le réel et le fictionnel. La deuxième consiste à assurer au texte une place sur le marché littéraire, monopolisé aujourd'hui par le roman; les autobiographies authentiques se faisant de plus en plus rares, l'auteur préfère mettre de côté l'aspect concret, formel et visible de sa vie pour, au contraire, se focaliser sur la réception de son texte, le lectorat étant friand des aventures de l'imaginaire. La dernière raison pour laquelle un auteur peut choisir une autofiction pour raconter sa vie consiste en le rôle de la mémoire dans ce genre d'entreprise. Ecrire l'histoire de sa vie inclut le fait de se souvenir du moindre détail, or dans la pratique, c'est quasiment impossible à faire ; la mémoire à un moment donné nous trahit, nous fait défaut, ne parvient plus à nous souffler les réminiscences du passé.

Pour Philippe Gasparini, l'autofiction n'est autre qu'une forme d'autobiographie post-moderne : "Disons que c'est une variante post-moderne de l'autobiographie, dans la mesure où elle ne croit plus à un discours historique cohérent, se fait reconstruction arbitraire et littéraire de fragments épars de mémoire. " ${ }^{(3)}$

En effet, dans l'autobiographie l'auteur s'engage à être sincère, à dire sa vie dans ses moindres détails, aussi fidèlement que possible, contrairement à l'autofiction où ce dernier admet et déclare qu'il peut y avoir oublis, modifications ou reconstructions de certains passages de sa vie, ce qui explique le fait que Gasparini parle de discours cohérent qui ne saurait être de pair avec l'autofiction ;cette dernière en appelle plutôt à un discours décousu, fragmenté qui traduirait l'état de confusion de l'auteur, obligé qu'il est de modifier certains aspects de sa vie.

C'est en 1977, à partir de son roman Fils et en réponse à Philippe Lejeune qui regrettait amèrement qu'il ne puisse y avoir dans la littérature un exemple 


\section{Quand l'auteur se livre : du vécu à la création littéraire Notes d'une musique ancienne de Salah Benlabed}

concret d'autofiction où l'auteur «fictiviserait» sa vie, que Serge Doubrovsy introduit la notion et néologisme d'autofiction qu'il définit comme suit : "L'autofiction, c'est la fiction que j'ai décidé, en tant qu'écrivain, de me donner à moi-même et par moi-même, en y incorporant, au sens plein du terme, l'expérience de l'analyse, non point seulement dans la thématique, mais dans la production du texte. ${ }^{(4)}$

En effet, non seulement et à travers cette définition, Serge Doubrovsky se fait lui-même écrivain et personnage-narrateur de son histoire fictive mais il ajoute un procédé inédit dans la fonctionnalisation de soi, il s'agit de l'analyse. Car, comme le démontre Doubrovsky, en rendant fictionnelle sa vie, l'auteur porte un autre regard sur ce qu'il est en observant ce qu'est le personnage qui lui ressemble tant et avec lequel il ne partage pas forcément les traits de caractère ni le parcours. C'est une sorte de distanciation que l'auteur peut effectuer à travers cette vie fictionnelle, entre la personne réelle qui produit l'histoire (l'auteur, lui-même) et le personnage-héros qui la vit ou plutôt la subit; cet effet-miroir (écrivain-personnage-héros) peut traduire tous les désirs refoulés, les actes indicibles, les paroles inavouables de l'auteur. L'analyse(ou plus exactement la psychanalyse) ne sera faite qu'à travers ce transfert de «personnalité » avec tout ce que cela peut comporter comme caractéristiques(idées, principes, valeurs, pensées...) dont le personnage-héros sera le porteur pour mieux « défendre » toute l'idéologie de l'auteur et mieux décrire sa psychologie .

En procédant ainsi, l'écrivain qui opte pour l'autofiction produit chez le lecteur, dans un incessant va-et-vient entre la personne réelle (l'auteur) et celle fictive, entre réalité donc et fiction, une véritable confusion, voire même un sentiment de contradiction puisque l'auteur n'a jamais vécu l'histoire qu'il relate; il en est cependant le rapporteur (narrateur) et l'acteur. Gérard Genette résume cet aspect "incohérent» de l'autofiction comme suit: "(le)pacte délibérément contradictoire propre à l'autofiction-Moi, auteur, je vais vous raconter une histoire dont je suis le héros mais qui ne m'est jamais arrivée-» ${ }^{(5)}$ En 1989, Vincent Colonna met en avant dans sa thèse L'autofiction, essai sur la fictionnalisation de soi en littérature, une définition de l'autofiction proche de celle qu'a proposée Serge Doubrovsky,en mettant l'accent sur ce personnage qu'invente l'auteur, qui peut porter le même nom que lui mais qui en est, cependant, différent par les aventures qu'il vit. Il la définit comme suit : « $L a$ fictionnalisation de soi consiste à s'inventer des aventures que l'on s'attribuera, à donner son nom d'écrivain à un personnage introduit dans des situations imaginaires. En outre, pour que cette fictionnalisation soit totale, il faut que l'écrivain ne donne pas à cette invention une valeur figurale ou métaphorique, qu'il n'encourage pas une lecture référentielle, qui déchiffrerait dans le texte des confidences indirectes. ${ }^{(6)}$ 
Cette projection de soi dans un univers fictionnel constitue, non seulement, tout un travail d'imagination mais aussi de finesse, de subtilité. En effet, l'auteur doit équilibrer son récit entre fiction et réalité de façon à ce que le lecteur continue à ressentir cette confusion propre à l'autofiction. De plus, il ne doit, en aucun cas, présenter des indices référentiels qui pourraient inciter et pousser le lecteur à faire un rapprochement avec la « véritable »vie de l'auteur.

Parmi les caractéristiques de l'autofiction les plus notables, et outre l'aspect psychanalytique évoqué ci-dessus, l'on citera également l'identité onomastique entre auteur-narrateur-personnage et la littérarité.

Le respect de l'identité onomastique de la triade auteur-narrateur-personnage est impératif dans l'écriture autofictionnelle. En effet, tout comme dans l'autobiographie, l'autofiction requiert de la part de l'auteur la régularité et la linéarité dans la situation narrative, stabilité assurée par un « je » commun entre auteur-narrateur-personnage; l'autofiction voudrait que ce soit la même personne qui écrit, raconte et vit l'histoire fictive; la différence entre autobiographie et autofiction ne résidant que dans la véracité des faits rapportés. L'auteur n'ayant le droit de tricher que sur le contenu, la forme (narrative du moins) doit être constamment portée par une seule et unique instance et ne doit en cas aucun cas être variable, flexible de sorte qu'on puisse l'appréhender ou l'expliquer à sa guise.

Contrairement à l'identité onomastique auteur-narrateur-personnage qui pourrait rapprocher l'autofiction de l'autobiographie, la littérarité souligne leurs différences du point de vue stylistique. La littérarité constitue tout le travail esthétique et formel qui se fait à l'intérieur d'un texte et auquel l'auteur s'adonne afin d'enjoliver son récit. Tout travail esthétique comporte des modifications, des ajouts, des occultations pratiques contraires à la définition même de l'autobiographie; la littérarité justifiant ainsi les problèmes de mémoire que peut rencontrer un auteur qui entreprend de rédiger son autobiographie. Celle-ci devant rester fidèle au vécu de l'auteur et à son parcours, toute modification altérerait donc l'interprétation référentielle que pourrait en faire le lecteur. En revanche, l'autofiction se définissant comme une histoire inventée mais dont l'auteur serait le héros, donc comportant déjà des transformations d'ordre thématique, la littérarité ne modifiera en aucun cas sa réception auprès du lecteur et relèvera au contraire toute l'inventivité et le style d'écriture de l'auteur. Le rapport au mot qu'a l'auteur, par le biais de la littérarité, se trouvera alors plus concret, plus tangible. En effet, le mot pris hors contexte et dans sa forme brute n'acquiert, chez l'auteur, de sens qu'une fois conçu, formulé et couché sur papier ; l'écriture autofictionnelle, se trouve ainsi être beaucoup plus l'aventure d'une écriture que l'écriture d'une aventure $^{(7)}$, beaucoup plus un travail de forme, un travail stylistique que le récit d'une vie fictive. 
Enfin il serait préférable de citer une des spécificités communes à l'autobiographie et à l'autofiction qu'a remarquée Philippe Gasparini.La psychanalyse ou l'analyse de soi en littérature passe par un travail d'introspection qui mène l'auteur à se revoir à travers l'imaginaire de son personnage ; personnage à travers lequel l'auteur fait partager ses souvenirs et son expérience au lecteur, tout ce travail de remémoration insuffle de l'émotion au texte et c'est ce que Gasparini appelle "l'investissement affectif des lieux de mémoire $\aleph^{(8)} ; c^{\prime}$ est, en quelque sorte, toute la charge émotionnelle et affective qu'un auteur peut mettre dans son récit et que le lecteur ressent à travers les souvenirs et les réminiscences de ce dernier; la mémoire (re)surgit déconstruite, les souvenirs affluent reconstitués, des bribes de vie recréés. L'auteur alors se dévoile, de son enfance à ses débuts de carrière, en passant par sa famille ou encore ses amis, il invite le lecteur à le connaître davantage en le plongeant dans son monde.

Gasparini remarque et déclare que l'affectivité relative aux lieux remémorés va souvent de pair avec la thématique de l'exil et du déracinement. En effet, le fait de s'expatrier, pour de multiples raisons, convoque souvent -si ce n'est de manière systématique- un travail mnémonique, et surtout si l'auteur décide d'écrire le récit de sa vie, que ce soit dans un pur esprit de vérité ou bien baigné dans la fiction. L'auteur, loin de son pays, se rappelle de son ancienne vie et revoit incessamment le chemin parcouru jusqu'à son arrivée en terre étrangère. Comme dans l'autobiographie, c'est un récit de nature rétrospective dans lequel l'auteur établit le bilan de sa vie; il revient sur ce passé qui le malmène (la culpabilité qu'il éprouve à avoir quitté le pays) et qui l'a mené sur les chemins de l'exil. Dans cette rétrospective, l'auteur essaye souvent de comprendre ce qui l'a poussé (outre les raisons qui lui paraissent évidentes) à laisser derrière lui toute sa vie ; ça sera alors toute son identité qui sera remise en question ; une identité éclatée, perdue qu'il essaiera de retrouver à travers l'écriture et la reconstitution de son passé.

C'est ce que nous avons relevé chez l'auteur que nous avons choisi : Salah Benlabed. Un auteur peu connu mais qui se révèle au lecteur, au fur et à mesure des pages, d'une manière touchante dans une autobiographie mêlée de fiction. Salah Benlabed réunit dans son récit Notes d'une musique ancienne ${ }^{(9)}$ aussi bien l'affectivité liée à la remémoration des lieux, que la thématique de l'exil et $\mathrm{du}$ déracinement. Quelques repères biographiques nous aideront à mieux cerner ce personnage et comprendre, par la suite, son choix quant à son récit nuancé de fiction.

Né à Mila, Salah Benlabed est issu d'une fratrie composée de trois enfants. Son père, avocat et descendant d'une grande famille de Constantine, et sa mère, originaire de la ville de Bejaïa, lui apprennent le goût de la lecture et plus tard le pousseront vers l'écriture. L'aînée de ses sœurs fut la première femme nommée juge de l'Algérie nouvellement indépendante; il s'agit de Farida 
Aberkane.Quant à la seconde ; c'est Leila Hamoutène, professeur de Français et écrivaine très impliquée dans la défense de la jeunesse. Parmi ses écrits : deux recueils de nouvelles Abîmes $^{(10)}$, Le Sablier ${ }^{(1)}$, un roman Sang et Jasmin $^{(12)}$, et un recueil de poèmes L'Enfant algérien ${ }^{(13)}$.

Suite aux multiples agressions liées à la décennie noire, Salah Benlabed et toute sa famille se réfugient à Constantine chez son grand-père, dans la grande maison familiale (située à l'ancienne adresse 89 , rue Combes) où il passera son adolescence, élève au lycée d'Aumale, actuel Redah Houhou.

Son baccalauréat obtenu, Salah Benlabed entame des études supérieures à Alger, à l'EPAU en architecture puis effectua le service national et son service civil dans le second bureau d'études d'Algérie dont il devient le Président Directeur Général. Il finira par démissionner de ce poste pour marquer son opposition à la politique de l'habitat. Il enseignera par la suite à l'école d'architecture d'Alger.

En 1988, il ouvre son propre bureau et réalise de nombreux projets de maisons individuelles dans le style traditionnel. Il participe également à la formation de jeunes dans ce domaine.

En 1994, il s'installe au Canada où il se met à l'écriture, qu'il considère comme une thérapie, et publie aux éditions La Pleine Lune plusieurs textes mettant en relief l'âme humaine et ses mystères, sa double appartenance identitaire, ce voyage qu'il propose au lecteur entre le passé et le présent, et toujours en filigrane la thématique de l'exil, qui est à la base de chacun de ses textes.

Salah Benlabed, en alliant autobiographie et fiction, laisse croire au lecteur qu'il lui raconte la stricte vérité, les évènements qui l'ont le plus marqués mais finit par avouer, quelques pages plus tard, qu'il a eu recours également à la fiction pour pousser encore plus loin le lecteur vers d'autres perspectives de recherches, d'autres interprétations ; l'alliance donc de l'autobiographie pure et de l'autofiction ouvre plusieurs champs de perception de ce que peut être la personne qu'est l'auteur.

La richesse d'un texte qui oscille entre l'autobiographie et l'autofiction réside dans la capacité de l'auteur à présenter sa vie, la décrire tout en l'enveloppant d'un voile "imaginaire»; il aura tendance peut-être à masquer ou rendre fictifs les évènements qu'il considère comme fâcheux ou qu'il préfère mettre de côté, voire même oublier. L'auteur en parlera alors de façon tacite, évoquant ses «mauvais » souvenirs en convoquant des personnages inventés de toutes pièces, ou alors en modifiant les personnes rencontrées ou qui l'auraient accompagné tout au long de sa vie, de manière à ce qu'elles soient conformes à l'image qu'il s'en est fait.

Dans Notes d'une musique ancienne, Salah Benlabed emprunte autant à l'autobiographie qu'à l'autofiction, son récit oscille donc entre réalité et fiction. L'auteur nous raconte le quotidien d'un expatrié (auquel on pourrait facilement 
l'identifier) qui a tout quitté et qui a tourné le dos à tout ce qu'il avait connu dans son pays pour fuir les menaces des intégristes ainsi que pour sauver sa fille de la barbarie qui y régnait, mais il sera vite déçu par son pays d'adoption, et par l'individualisme qui caractérise ses habitants. L'auteur en présentant son texte au lecteur lui donne à croire que l'histoire qu'il contient est la sienne, qu'il l'a bien vécue, qu'elle est supposée donc être réelle, et qu'il en est le rapporteur.

C'est au cours des premières pages de son texte que Salah Benlabed avertit le lecteur et l'assure de la véracité de ce qui va suivre en s'exprimant au « je ».La narration est donc portée par une seule et même personne, qui n'est autre que l'auteur. Il est donc tout à fait légitime que le lecteur croit qu'il a affaire à une autobiographie, d'autant que toutes les autres caractéristiques propres à ce type d'écriture sont apparentes dans le texte. En effet, Notes d'une musique ancienne réunit les trois premières critères de l'autobiographie, et dont a fait mention Philippe Lejeune dans sa définition, à savoir : le récit rétrospectif et l'écriture personnelle, la vie individuelle.

L'autobiographie est par définition le récit d'une vie, des évènements et des personnes qui ont eu un impact considérable sur la personnalité de l'auteur; de ce fait un travail mnémonique est impératif à ce type d'entreprise. L'écrivain évoquera son passé, ses moments de bonheur, ses états d'âme ou encore ses tourments. Il replongera dans son passé pour ainsi se confesser plus librement au lecteur qui percevra par la suite sa personnalité et un peu plus sa psychologie.

Salah Benlabed use de ce procédé dans son texte; tout en parlant de sa vie d'expatrié et en se présentant au lecteur de manière directe et concrète, il fera quelques haltes sur le chemin parcouru depuis sa terre natale jusqu'à son exil et celui de sa fille. L'auteur évoque son passé glorieux, ponctué de retrouvailles familiales, des longues siestes d'été bercées par les interminables histoires dignes des Milles et une nuit que leur contait sa cousine. Il raconte également l'ambiance qui régnait dans la maison de son grand-père, havre de paix et de réjouissances, auxquelles participait avec joie sa grand-mère ; une personne dont était très proche l'auteur et avec laquelle il entretenait une relation fusionnelle. L'auteur finit par présenter au lecteur un tableau des plus reluisants de cette époque-là, désormais révolue, tout en dressant les portraits de tous les membres de sa famille.

Toutes ces réminiscences sont présentées en prose comme l'exige l'écriture autobiographique. Cette forme d'écriture, et donc de narration assure la continuité et la régularité des faits rapportés. Néanmoins, et même si l'on pourrait croire que les incessants va-et-vient entre le passé et le présent pourraient troubler la linéarité de la narration, il n'en est rien puisque l'écriture du souvenir viendrait justifier ce choix-là de la part de l'auteur car récit rétrospectif et prose vont souvent de pair. Salah Benlabed, en optant pour la 
prose et même s'il convoque le passé dans son récit, assure la fluidité de sa narration indispensable à la compréhension du texte.

Nous savons entre autre qu'écrire son autobiographie relève d'une expérience personnelle dans laquelle l'auteur se met en avant et se dévoile : il parle en son nom de sa propre vie. Ici vient se concrétiser l'aspect personnel de l'écriture portée par le «je ».Salah Benlabed se confie dans son texte à travers ce même « je » censé refléter trois entités : l'auteur, le narrateur et le personnage. Il est l'auteur, le narrateur et l'un des personnages de l'histoire qu'il raconte. L'identité onomastique est donc respectée mais dans ce cas, ce «je» est foncièrement anonyme. Cet anonymat traduit l'identité éclatée et menacée du personnage-narrateur, et du coup celle de l'auteur aussi, perdu qu'il est entre son pays d'accueil et sa terre natale, il n'appartient à aucun des deux puisqu'il a tourné le dos à son Algérie et son pays d'adoption ne l'intègre pas non plus à sa société. Cet anonymat peut être aussi une façon de pousser le lecteur à s'identifier à ce narrateur et ainsi vivre son expérience pour mieux comprendre son vécu. Ce «je» anonyme dont l'auteur ne fait aucune description n'empêche nullement le caractère personnel de l'écriture; l'auteur voulant avant tout raconter son vécu. Prenons pour exemple les innombrables monologues qui ponctuent le récit et qui se font à travers ce "je» toujours insaisissable pour le lecteur mais qui se confie malgré tout et qui parle de tous les maux et les malaises engendrés par l'exil. Salah Benlabed, en gardant anonyme son personnage-narrateur laisse percevoir une époque douloureuse de sa vie: celle où sa mère, atteinte d'Alzheimer ne le reconnait pas, elle l'affublera donc de manière indirecte du sobriquet de Personne. Même si ce surnom est lié à sa maladie, le personnage-narrateur ne peut s'empêcher d'y lire un message : celui qui évoque la rupture avec sa mère et toute sa vie d'antan, il n'est le fils de personne; l'auteur nous dresse le portrait d'une personne solitaire et torturée par les souvenirs du passé et les tourments d'un présent alourdi par les remords et les regrets.

Face à l'anonymat du personnage-narrateur et afin de vérifier l'authenticité du récit, faire appel aux opérateurs d'identification du héros avec l'auteur semble indispensable. Le parcours du personnage-narrateur et de l'auteur sont identiques dans le sens où tous deux ont fui la violence qui sévissait dans leur Algérie natale pour venir "survivre » dans un pays qui leur est étranger. Les regrets et la déception ont succédé à l'enthousiasme et à l'euphorie des premiers jours. Dans cette expédition vers l'inconnu, le personnage-narrateur entraine avec lui sa fille (ce que fait l'auteur également) afin de lui assurer un avenir beaucoup plus prometteur que celui qu'elle aurait pu avoir en Algérie.

Salah Benlabed parle de sa famille, de ses parents et surtout de sa grand-mère. L'auteur nous avouera dans un entretien qu'il avait un rapport privilégié avec cette dernière, qu'il passait beaucoup de temps ensemble. Quant à ses parents, il nous les présente dans le texte mais aussi «en vrai » comme étant des 
personnes d'une sagesse et d'une loyauté incomparables. Cependant le personnage-narrateur ainsi que l'auteur assistent avec impuissance à la métamorphose et à la déchéance de ces deux êtres qui leur sont si chers. En effet, la mère atteinte d'Alzheimer ne reconnait plus son fils(d'où le fait qu'il se sente sans identité, comme déraciné),et le père après avoir souffert de sa radiation du Barreau pour activisme politique par l'administration française, finit par être cloué au lit par la maladie, et ce jusqu'à sa mort. Ce père qui était à la fois présent par l'éducation rigoureuse et stricte qu'il a donnée à ses enfants, et absent par les barrières qu'il a établi, ne laissant jamais la porte ouverte aux épanchements affectifs. Le personnage-narrateur ne s'étonne donc pas de se voir reproduire le même schéma avec sa fille, donnant l'image d'un père froid et distant, cet aspect-là s'étant vite effrité au contact de l'exil.

Concernant la profession de Salah Benlabed et du personnage-narrateur, Gasparini déclare que dans une autobiographie, l'écrivain pourrait donner au personnage-narrateur le même métier que le sien ou du moins s'en rapprochant. C'est ce qui se passe dans Notes d'une musique ancienne, l'on sait que Salah Benlabed est connu comme écrivain, beaucoup plus au Canada, c'est donc tout naturellement qu'il donne à son personnage-narrateur le métier de journalistereporter.Ecrivain et reporter étant deux métiers qui se basent sur le témoignage et la documentation et trouvent donc racine dans la rédaction. Ce trait commun assure un rapport étroit entre personnage-narrateur et auteur et présente le caractère original et véridique du récit. En raison de son métier, et au vu du contexte social sur lequel s'ouvre le récit et qui n'est autre que la décennie noire, le personnage-narrateur se verra incarcéré pour outrage à la nation et injure à l'Etat. L'auteur qui a eu aussi à vivre ce moment difficile, lors d'un de ses séjours en Algérie nous décrit son personnage au sein de la prison, ses compagnons et son geôlier. Il a donc fait vivre son expérience au personnagenarrateur pour témoigner des conditions dans lesquelles sont enfermés les prisonniers et la manière avec laquelle ils sont traités, c'est en quelque sorte, un témoignage sur le milieu carcéral.

Tous ces traits communs à Salah Benlabed et à son personnage-narrateur tendent à indiquer au lecteur que ce récit est purement autobiographique. Or, en ayant bien à l'esprit les repères biographiques dont nous avons bénéficié, nous remarquons qu'il y a certaines «absences», des modifications et des changements susceptibles de fausser l'interprétation du lecteur et de l'induire en erreur. Ces transformations et ces oublis relèvent de l'emploi de l'autofiction qui n'est autre que la fictionnalisation de certains aspects de la vie de l'auteur. Cette dernière réside dans le texte choisi, à travers le fait de s'inventer un frère ; or nous savons que Salah Benlabed n'a que deux sœurs. Le fait de s'inventer un frère est une stratégie d'écriture de la part de l'auteur, ce frère étant en fait que le reflet de la société algérienne qui a fraîchement acquis l'indépendance; une société cupide et âpre au gain. Ce trait apparait dans le 
texte au moment où le personnage-narrateur (et aussi l'auteur) perd sa mère : rentré au pays pour son enterrement, il ne réclame de son frère que les photos de la défunte alors que ce dernier se préoccupe plutôt du partage des biens.

L'auteur s'invente un frère mais ne parle à aucun moment de son fils lui préférant sa fille, ne voulant garder dans son texte que des figures féminines, lui qui n'a été élevée pratiquement que par des femmes et auxquelles il voue un respect et une admiration sans failles. Le fait qu'il ait choisi de mettre sa fille en avant et d'écarter son fils traduit également son envie d'insuffler dans son récit un peu plus d'émotion et d'affectivité, voulant de cette façon casser le stéréotype qui prime sur la mentalité maghrébine, et qui veut qu'un père soit distant et froid avec ses filles.

Dans un des passages du texte, Salah Benlabed évoque le souvenir du cadeau de mariage offert par sa mère lors de son installation dans sa nouvelle maison, il s'agissait d'un figuier, que le personnage-narrateur a découvert un matin, arraché et déraciné. C'est une image symbolique, une métaphore qui représente la rupture de l'auteur (et du personnage-narrateur) avec ses racines, ses ancêtres et sa patrie. La fictionnalité de ce passage réside dans le fait que l'auteur ait quelque peu modifié le type d'arbre offert par sa mère, en effet il s'agissait d'un palmier et non d'un figuier. Ce choix se justifie par le fait que l'auteur voue un attachement sans précédent au désert de son Algérie, mais qu'au contact de l'exil et de ce pays étranger, l'image symbolique qu'il représentait s'est effritée et de ce fait, n'a fait qu'accroitre la souffrance du personnagenarrateur.L'auteur a donc voulu préserver ,de son monde extérieur et tellement hostile(ce pays d'exil), ce que ce palmier représentait pour lui, ce qu'il avait de plus significatif et de plus poignant au vu de sa désertion. Voulant garder tout ancré au fond de lui l'image et le reflet de son pays et de ses ancêtres à travers cet arbre fétiche, l'auteur a donc décidé d'user de stratégie autofictionnelle et d'effectuer certaines modifications pour véhiculer tout au long du récit toute la charge émotionnelle liée à cet épisode de sa vie.

Concernant son entourage et particulièrement son épouse, le personnagenarrateur raconte dans le texte qu'elle a péri dans un accident de voiture, lors d'une escapade effectuée au désert mais en vérité, l'épouse de Salah Benlabed est bel et bien vivante. Le fait de l'avoir totalement écartée du récit révèle l'envie qu'avait l'auteur d'écrire un texte dans lequel seule sa fille devait être sa « campagne de route ».Il lui concède ainsi une place privilégiée puisqu'elle partagera avec lui les moments les plus difficiles dans le pays d'accueil, jusqu'à son retour en Algérie. En éliminant donc sa femme qui représentait le passé, son ancienne vie, l'auteur évoque la possibilité d'une vie ailleurs qu'au désert (la terre natale) et différente (mais non moins angoissante) que celle laissée là-bas, en Algérie. Ainsi il aura sacrifié sa femme, son passé pour s'ouvrir à un avenir avec sa fille, plus apaisant que tout ce qu'il a pu vivre jusqu'à présent. En plus du personnage incarné par sa fille, l'auteur fait appel 


\section{Quand l'auteur se livre : du vécu à la création littéraire Notes d'une musique ancienne de Salah Benlabed}

dans son récit à une autre figure féminine, un personnage instable et étrange, rencontré en terre d'exil et surnommé "la fée des lundis ».Cette rencontre représente en fait la tentative d'intégration du personnage-narrateur au sein d'une nouvelle société. Tentative qui s'est soldée par un échec au vu des innombrables critiques et remarques désobligeantes dont elle le gratifiait, lui rappelant toujours ses origines, sa désertion et son abandon. La fée des lundis finira par disparaitre le laissant seul face à ses angoisses et ses remords. Ce qui sera pour lui bénéfique puisqu'elle l'avait détourné de tous ses projets et de tous ses rêves, l'obligeant à changer de comportement, de personnalité, lui qui lui accordait tant d'importance et d'intérêt, croyant qu'il pourrait, à travers elle, être considéré non plus comme un étranger mais bien comme un citoyen ayant fait ses preuves, en réussissant son intégration.

Tous ces changements et ces ajouts donnent un cachet fictionnel au texte et l'auteur ne s'en cache pas ; son écriture oscille ainsi entre l'autobiographie, des faits qui se sont réellement produits, des personnes qui existent vraiment, et l'autofiction et tout le travail créatif qu'elle convoque et qui en découle, d'où la richesse de ce type d'écritures qui laissent voir au lecteur deux pans de la personnalité de l'auteur.

\section{Conclusion}

Enfin, et pour comprendre la place que tiennent aujourd'hui les écritures autobiographiques (ainsi que toutes les variantes qui en découlent), il serait judicieux de les remettre dans leur contexte d'apparition. Ce type d'écriture n'a pas de tout temps été bien accueilli par les critiques littéraires. L'autobiographie et l'autofiction entre autres, n'ont donc pas été définies comme genre majeure, et de ce fait, ont été considérées comme « un genre pas très sérieux » selon Marie Darrieussecq ou encore carrément comme « mauvais genre » selon Lecarme.En effet pour la critique, l'élaboration d'une œuvre telle que la conçoit la littérature doit passer par plusieurs étapes, qu'on pourrait définir comme théoriques ; le fait de raconter les innombrables évènements qui peuvent marquer une vie serait une entorse à la règle. L'écriture ne devrait pas être empreinte du « bagage personnel » de l'auteur, et si c'est le cas, le lecteur doit le percevoir de manière subtile; or l'autobiographie et l'autofiction n'obéissent pas forcément à ce schéma.

C'est à partir du XVIe siècle et de la montée de l'humanisme que le genre prend, plus ou moins, forme avec cet intérêt soudain porté à l'individu et au Moi. Ce dévoilement de soi avait commencé avec les mémoires, les essais et ce travail a cheminé vers Les Confessions (authentique autobiographie) au XVIIIe siècle. Depuis cette date, l'autobiographie considérée comme la forme pionnière de l'écriture personnelle, intimiste s'est vue varier et prendre d'autres formes qui, soulignons-le, partent du même principe (le vécu de l'auteur):l'autobiographie fictive, le roman autobiographique, l'autofiction, l'autofabulation..., etc. 
De par la complexité de l'autobiographie et de l'autofiction, la difficulté de définir toutes leurs variantes, de décrire et de comprendre tous les procédés qui permettent leur production, mais du fait aussi qu'elles abordent toutes la même thématique, qu'elles partagent la même acception et caractéristiques(à quelques points près), nombres de critiques littéraires dont Philippe Gasparini,Philippe Lejeune, Jacques Lecarme,Serge Doubrovsky ou encore Vincent Colonna, pour ne citer qu'eux, se sont penchés dessus et s'y sont intéressés au point de leur consacrer leurs travaux de recherches et nombres d'articles.

Genres initialement dénigrés au profit de la production romanesque et poétique, l'autobiographie et l'autofiction, entre autres, ont fini par acquérir une importance majeure dans la littérature ; car c'est à travers l'écriture de soi que le lecteur peut cerner au mieux le personnage qu'est l'auteur, et pour comprendre un texte, ne doit-on pas connaitre qui en est le " producteur»?

En effet, l'écriture autobiographique et autofictionnelle permet au lecteur de faire immersion dans l'univers aussi bien réel qu'imaginaire de l'auteur, ce qui peut l'amener à le percevoir sous deux angles différents et totalement opposés mais qui font partie de tout un chacun, et c'est ce qui donne une autre vision de la personnalité et du caractère de chaque écrivain. La richesse et la multiplicité d'interprétations que peut offrir ce genre d'écritures sont donc d'une importance majeure dans la perception et la compréhension de la personnalité de l'auteur. L'écriture du Moi, ce texte baigné de confidences et de confessions devient alors pour le lecteur, une sorte de texte documentaire, de fiche représentative de l'auteur et de son vécu, elle acquiert un tout autre sens par rapport au message que peut véhiculer un roman et dont la finalité serait bien différente de celle que peut comporter une autobiographie ou une autofiction; ces dernières relèveraient donc plutôt du témoignage et de "l'information », l'auteur voulant se confier au lecteur et l'inviter à partager ,bien plus que des aventures qui se succèdent au fil des pages, des passages de son existence. Ces petits morceaux de vie éclaireront, à leur tour, les textes ultérieurs que l'auteur peut proposer à son lectorat, et qui souvent entretiennent d'étroits rapports avec le texte «fondateur». Se référant ainsi aux innombrables indices biographiques recueillis ça et là, à travers l'autobiographie ou l'autofiction que peut présenter l'auteur, le lecteur finira par établir le fil conducteur de toute l'œuvre de ce dernier, ce qui lui permettra d'avoir une vision globale de toute sa personne.

Bibliographie

-Benlabed, Salah, Notes d'une musique ancienne, éditions La Pleine Lune(Canada) 2007, éditions APIC (Algérie) ,2010.

-Lejeune, Philippe, Le Pacte Autobiographique, Paris, éditions Seuil, collection «Points », 1975, nouvelle édition 1996.

-Gasparini, Philippe, Est-il je, Roman autobiographique et Autofiction, Paris, éditions Seuil, collection « Poétique »,2004. 


\section{Quand l'auteur se livre : du vécu à la création littéraire Notes d'une musique ancienne de Salah Benlabed}

-Gasparini, Philippe, Autofiction, une Aventure du langage, Paris, éditions Seuil, collection « Poétique »,2008.

-Doubrovsky, Serge, « Autobiographie /Vérité/Psychanalyse », Paris, éditions PUF, 1998.

-Genette, Gérard, Fiction et Diction, Paris, éditions Seuil, collection «Poétique »,2008.

-Colonna, Vincent, L'Autofiction, essai sur la fictionnalisation de soi en littérature, Paris, EHESS, 1989.

-Ricardou, Jean, Problèmes du nouveau roman, essais, Paris, éditions Seuil, collection « Tel Quel»,1967.

Note :

1- Le Pacte Autobiographique, Philippe Lejeune, éditions Seuil, 1975, nouvelle édition 1996, coll. «Poétique », Paris, p.14

2- Est-il je, Roman autobiographique et Autofiction, Philippe Gasparini, éditions Seuil, coll. «Poétique », Paris, 2004, p.25.

3- Autofiction, une Aventure du langage, Philippe Gasparini, éditions Seuil, coll. « Poétique », Paris, 2008, p.221

4-« Autobiographie/Vérité/Psychanalyse »Serge Doubrovsky, éditions PUF, Paris, 1988, p.77

5- Fiction et Diction, Gérard Genette, éditions Seuil, coll. « Poétique », Paris, 2008, p.221

6- L'Autofiction, essai sur la fictionnalisation de soi en littérature, Vincent

Colonna, EHESS, Paris, 1989, p.10

7-Jean Ricardou, Problèmes du nouveau roman, essais, éditions Seuil, coll. « Tel Quel», Paris, 1967, p.111

8-Est-il je, Roman autobiographique et autofiction, Philippe Gasparini, éditions Seuil, coll. « Poétique », Paris, 2004, p.47

9- Salah Benlabed, Notes d'une musique ancienne, éditions La Pleine Lune (Canada), 2007, éditions APIC (Algérie) ,2010

10- Abîmes, Leila Hamoutène, ENAG éditions, 1992

11-Le Sablier, Leila Hamoutène, ANEP éditions, 2002

12- Sang et jasmin, Leila Hamoutène, MARSA éditions, 2000

13-L'enfant algérien, Leila Hamoutène, éditions UNICEF, Alger, 2002 